\title{
Queues in Health
}

\section{J. B. F. Adan · Richard J. Boucherie}

Published online: 12 December 2014

(C) European Union 2014

The aim of this special issue of Queueing Systems is to highlight the role of queueing theory in sustaining and improving the quality of care in our health care system. On one hand, it aims at showing the possible impact of queueing theory on optimisation in health care. On the other hand, it aims at drawing health care to the attention of queueing theorists to further increase the number of contributions advancing the mathematical treatment and stochastic analysis of queues in the health care domain.

There is great need for a thorough mathematical analysis of health care systems. Health care expenditures are rapidly growing and in western countries may increase from $10-15 \%$ of GDP in 2015 to roughly $20-25 \%$ in 2035 under the current resource allocation policies. This increase is partly due to improved medical techniques and devices, but to a large extent due to our ageing population. Sustaining the current quality of care seems to require a rapidly increasing work force, which is in stark contrast with the reducing workforce due to ageing. Operations research allows us to maintain or increase the current quality of care for a growing number of patients without increasing the required resources. Efficient planning of operating theatres will reduce the wasted hours of staff, optimising patient admission and allocation of resources will reduce waiting times, balancing the number of patients in wards will reduce peaks, and therefore increases the efficiency of nursing care and efficient rostering of staff allows for more work to be done by the same number of people.

\footnotetext{
I. J. B. F. Adan

Department of Mechanical Engineering, Eindhoven University of Technology, P.O. Box 513, 5600 MB Eindhoven, The Netherlands

e-mail: iadan@tue.nl

\section{R. J. Boucherie $(\varangle)$}

Stochastic Operations Research, Department of Applied Mathematics, Faculty of Electrical Engineering, Mathematics, and Computer Science, University of Twente, P.O. Box 217, 7500 AE Enschede, The Netherlands

e-mail: r.j.boucherie@utwente.nl
} 
As many of the encountered problems involve delay due to randomness, queueing theory seems to be a natural candidate to tackle these problems. Employing queueing theory is not only targeted to improving efficiency, since improved efficiency also leads to increased job satisfaction as experienced work load is often dominated by those moments at which the work pressure is very high, and it also improves patient safety since errors due to peak work load will be avoided.

Fortunately, more and more health care organisations are realising that mathematical operations research methods may provide an approach to sustain our health care system. Such an approach requires close collaboration between mathematicians, operations research specialists and health care professionals to tackle resource optimisation challenges of the health care sector and implement their solutions in health care organisations. Currently only a few percent of the queueing theory specialists are working in health care, which is not enough to alleviate the pressure on the health care work force. The main body of papers that analyse queues in health care apply existing queueing results. It seems that this may be enough for the current state of affairs in health care. However, to further advance the opportunities for optimisation, new methods on the boundary of queueing theory and health care are of utmost importance. This special issue contains a collection of four papers at this intersection. A brief preview of the contents of the contributions follows.

From a queueing perspective, the focus of the contributions of this special issue is on the intersection of prioritisation and appointment scheduling in queues.

Kuiper, Kemper and Mandjes consider the phase-type counterparts of the servicetime distributions in an appropriately chosen $D / G / 1$ queue to obtain appointment schedules that balance the interests of the patients (low waiting times) and the medical staff (low idle times) via two approaches: arrival epochs of all patients are either simultaneously chosen, or the arrival epoch of a next patient is scheduled given the scheduled arrival epochs of all previous patients. Various aspects of both approaches are investigated through systematic numerical experiments.

Zonderland, Boucherie and Al Hanbali consider prioritisation of patients on care pathways in outpatient clinics. These patients require an appointment slot in a time window, making sure that subsequent steps in the pathway can also be accommodated. They consider the blocking probability of priority patients in a discrete-time queue, where priority patients reserve a time slot in a time window and are blocked and cleared when all slots in the required time window are occupied by other priority patients. This discrete-time queue is modelled as a $G e o^{x} / D / 1$ queue, the equilibrium probability vector of which appears to be matrix geometric.

Luo, Kulkarni and Ziya develop a two-queue tandem model for an appointmentbased service system: an appointment queue followed by a service queue. The particular feature in this system is that a patient that experiences a long waiting time in the appointment queue may become a no-show for the service queue with probability depending on the time spent in the appointment queue. Results include server utilisation and patient long-run average waiting times in both queues.

Drekic, Stanford, Woolword and McAlister introduce a level-dependent quasibirth-and-death process for patients' waiting times for organ allocation. Organ allocation is typically done on the basis of the health status of the patient. Most patients arrive as regular patients, and may decide to abandon the wait list or are promoted to 
priority patients as the health of a patient degrades while waiting. The model is calibrated using liver transplantation wait list data, provided by a regional health centre in Canada.

Finally, the above collection of four papers has been chosen for their quality and relevance to this special issue after a highly selective refereeing process. We would like to thank the authors of all submitted papers and the referees for their interest and their support for this special issue of Queueing Systems. 